\title{
BMJ Open Allogeneic human umbilical cord- derived mesenchymal stem/stromal cells for chronic obstructive pulmonary disease (COPD): study protocol for a matched case-control, phase I/II trial
}

Duc M Hoang (D) , ${ }^{1}$ Kien T Nguyen, ${ }^{2}$ Anh H Nguyen, ${ }^{3}$ Bach N Nguyen, ${ }^{3}$ Liem Thanh Nguyen ${ }^{4}$

To cite: Hoang DM, Nguyen KT, Nguyen AH, et al. Allogeneic human umbilical cordderived mesenchymal stem/ stromal cells for chronic obstructive pulmonary disease (COPD): study protocol for a matched case-control, phase $1 /$ II trial. BMJ Open 2021;11:e045788. doi:10.1136/ bmjopen-2020-045788

- Prepublication history for this paper is available online To view these files, please visit the journal online (http://dx.doi org/10.1136/bmjopen-2020045788).

Received 13 October 2020 Revised 16 April 2021 Accepted 23 April 2021
Check for updates

(C) Author(s) (or their employer(s)) 2021. Re-use permitted under CC BY-NC. No commercial re-use. See rights and permissions. Published by BMJ.

For numbered affiliations see end of article.

Correspondence to

Dr Duc M Hoang;

v.duchm3@vinmec.com

\section{ABSTRACT}

Introduction The global prevalence of chronic obstructive pulmonary disease (COPD) is increasing, and it has become a major public health burden worldwide, including in Vietnam. A large body of preclinical and clinical studies supports the safety of mesenchymal stem/stromal cells (MSCs) in the treatment of lung injury, including COPD. The aim of this trial is to investigate the safety and potential therapeutic efficacy of allogeneic administration of umbilical cord-derived MSCs (UC-MSCs) as a supplementary intervention in combination with standard COPD medication treatments in patients with moderate-to-severe COPD based on the Global Initiative for Chronic Obstructive Lung Disease (GOLD) 2019 and Vietnam Ministry of Health's guidelines.

Methods and analysis This matched case-control phase V/ II trial is conducted at Vinmec Times City International Hospital, Hanoi, Vietnam between June 2020 and December 2021. In this study, 40 patients will be enrolled and assigned into two age-matched, gender-matched and COPD condition-matched groups, including a UC-MSC group and a control group. Both groups will receive standard COPD medication treatment based on the GOLD 2019 guidelines and the Vietnam Ministry of Health protocol. The UC-MSC group will receive two doses of thawed UC-MSC product with an intervention interval of 3 months. The primary outcome measures will include the incidence of prespecified administration-associated adverse events and serious adverse events. The efficacy will be evaluated based on the absolute changes in the number of admissions, arterial blood gas analysis, lung function and lung fibrosis via CT scan and chest $X$-ray. The clinical evaluation will be conducted at baseline and 3, 6 and 12 months postintervention.

Ethics and dissemination Ethical approval was secured from the Ethical Committee of Vinmec International Hospital (number:166/2019/QĐ-VMEC) and Vietnam Ministry of Health (number:2002/QĐ-BYT). The results will be reported to trial collaborators, publication in peerreviewed academic journals.

Trial registration number NCT04433104.

\section{INTRODUCTION}

Chronic obstructive pulmonary disease (COPD) is described-but not defined-as

\section{Strengths and limitations of this study}

- This project is the first matched case-control phase I/II study to evaluate the safety and efficacy of allogeneic administration of umbilical cord-derived mesenchymal stem/stromal cells (UC-MSCs) as supplementary treatment in combination with standard medication treatments for patients with moderate-to-severe chronic obstructive pulmonary disease (COPD).

- To address the challenge of evaluating the effectiveness of MSC treatment in COPD by using quantitative and qualitative research methods.

- To interlink the treatment effectiveness with stem cell phenotype analysis to broaden our understanding of UC-MSC effects in COPD.

- The limitation of this study is that it was not conducted as a randomised control trial due to the complexity of the process and patient recruitment as well as the challenges of undertaking clinical trials in patients with COPD due to the heterogeneity of disease mechanisms and phenotypic expression.

one of the major chronic lung diseases characterised by persistent and progressive airflow obstruction. It is caused by an elevated chronic pulmonary inflammatory response in the airways and bronchial structure to noxious particles or gases. The pathological hallmarks of the disease include obstructive bronchiolitis, emphysema and mucus hypersecretion. ${ }^{1}$ Despite many medical advancements and technological improvements, our understanding of the pathological mechanisms underlying the progressive and detrimental development of COPD remains incomplete, the definition of the disease is controversial, diagnostic tests are inaccurate and unstandardised, and the treatment is inadequate. ${ }^{2}$ A recent report stated that the global prevalence of COPD increased by $44 \%$ within the 
last 20 years, and more than 3.2 million patients died each year from COPD worldwide (accounting for approximately $5 \%$ of all deaths globally per year). ${ }^{3}$ In Vietnam, according to the WHO report, $7.1 \%$ of males and $1.9 \%$ of females aged 40 and above are diagnosed with COPD. Consequently, approximately $25 \%$ of hospital beds in respiratory wards are required for patients with COPD, resulting in a heavy burden to Vietnamese Medical Infrastructure and reducing patients' health and quality of life. ${ }^{4}$ The current pharmacological medications for COPD include the use of inhaled bronchodilator drugs, such as long-acting $\beta$ agonists and long-acting muscarinic antagonists, the use of inhaled corticosteroids or a combination of these medications. Although it is generally accepted that pharmacological interventions via inhalation would allow the accurate delivery of drugs and increase the clinical benefits, incorrect inhaler technique and a lack of adherence when feeling healthy caused worse dyspnoea, impaired health condition and increased the frequency of exacerbations and hospitalisations in Vietnamese patients with COPD. ${ }^{5}$ Therefore, identifying novel effective therapies for patients with COPD is urgent and important.

Since their first discovery in 1968, mesenchymal stem/ stromal cells (MSCs) have been intensively studied because of their therapeutic and regenerative features. The nomenclature of MSCs has been debated recently due to not only the biological features of the MSCs themselves but also the medical abuse of the term 'stem cells' inferring direct medical benefit. ${ }^{6}$ To standardise the characterisation of MSCs and facilitate their therapeutic implications, the International Society for Cellular Therapy (ISCT) has proposed the minimum criteria to define human MSCs. ${ }^{78}$ In our study, MSCs were defined as MSCs, which are a class of adult mesenchymal progenitor cells derived from either bone marrow, adipose or UC tissue and met the minimum criteria of ISCT. Among various sources of MSCs, human UC-derived MSCs (hUC-MSCs) are potentially more advanced than their adult counterparts (bone marrow or adipose) for several reasons: (1) ease of collection as it is a noninvasive process, (2) waiving ethical barriers as UC is medical waste discarded at birth, (3) rapid proliferation rate, (4) maintenance of normal karyotype during prolonged culture in vitro and (5) higher paracrine potency than adult tissue-derived MSCs. ${ }^{9}$ The therapeutic potential of hUC-MSCs has been proven in clinical studies, especially animal pulmonary disease models, including acute respiratory distress syndrome (ARDS), bronchopulmonary dysplasia (BPD) and COPD. It has been reported that UC-MSCs are effective in reducing lung inflammation and fibrosis processes, preventing secondary infection, decreasing immune system damage, increasing bronchoalveolar fluid clearance and enhancing the regeneration of alveolar epithelium layers. ${ }^{10-12}$ The majority of intravenously administered MSCs reportedly remain in the lung, especially pulmonary microvessels, which potentially contribute to their beneficial effects in pulmonary disease models. ${ }^{13}$ Hence, the safety and therapeutic effects of
UC-MSC administration for COPD require further investigation and clarification.

To date, five completed clinical trials have used bone marrow mononuclear cells (BMMCs), bone marrowderived MSCs (BM-MSCs) and UC-MSCs in COPD. ${ }^{14-19}$ Although these clinical trials provided an enormous amount of data supporting the safety of the therapy in the treatment of COPDs, the efficacy of the treatments remained controversial due to several limitations, including trial design, lack of standardisation of cell numbers administered to patients, timing of MSC administration, and most importantly, the lack of a control group in several studies. Moreover, the variations in patient selection based on the severity and stage of COPD could be attributed to the effectiveness of the cell therapy, resulting in caution in data interpretation. Last but not least, the quality of administered MSCs also plays a significant role in the effectiveness of the treatment, that is, the status of the cells (fresh culture vs. frozen cells), cell sources (from young healthy donors or aging individuals), dosage frequency, etc. Therefore, identification of the potential sources of MSCs (such as UC-MSCs), larger sample size with matched controls, and standardised classification of COPD using international accepted criteria is required to further investigate the safety and efficacy of MSC therapy. Based on preclinical studies and previous promising findings, we designed a matched control phase I/II clinical trial to evaluate the safety and potential efficacy of the intravenous infusion of allogeneic hUC-MSCs in patients with COPD characterised based on the Global Initiative for Chronic Obstructive Lung Disease (GOLD) 2019 criteria. $^{20}$

\section{METHODS AND ANALYSIS \\ Study objectives}

The aim of this trial is to evaluate the safety and potential efficacy of allogeneic UC-MSC administration in patients with COPD. There are three specific objectives:

1. Evaluate the safety and potential therapeutic effectiveness of intravenously (IV) administered UC-MSCs in patients with COPD.

2. To prove the hypothesis that IV administration of UCMSCs can improve lung function and reduce inflammatory responses in the lungs and fibrosis.

3. Explore the potential therapeutic mechanism of UCMSCs in the treatment of COPD.

\section{Study design and ethics}

This matched case-control phase I/II clinical trial was approved by the Ethical Committee of Vinmec International Hospital (number: 166/2019/QĐ-VMEC) and Vietnam Ministry of Health (number: 2002/QĐ-BYT). To achieve the aims, a total of 40 patients with COPD will be recruited at the Internal Medicine Department at Vinmec Times City International Hospital, Hanoi, Vietnam, between June 2020 and December 2021. A flow chart of the study design is shown in figure 1 . 


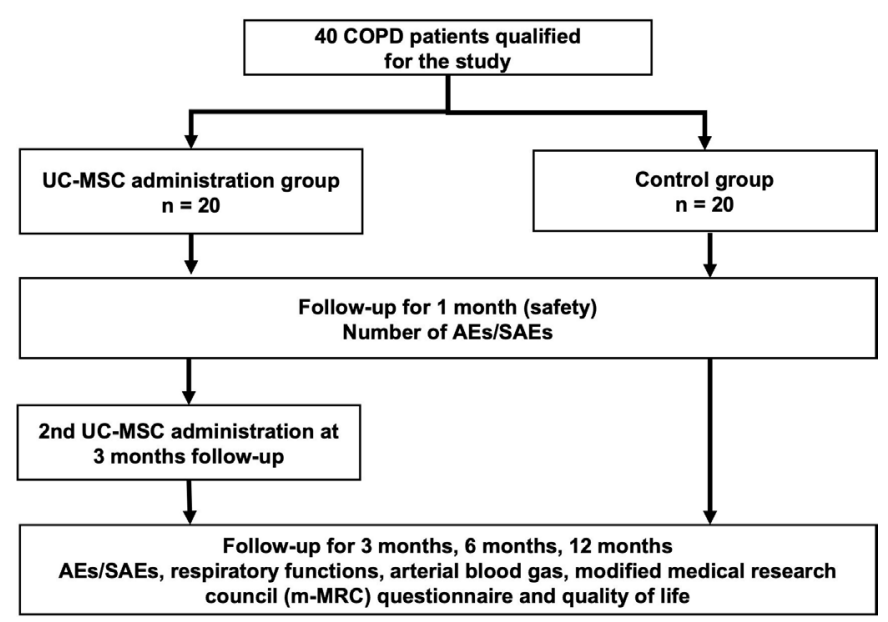

Figure 1 Schematic of the study. Patients with COPD will be screened to enrol in the study. Patients from the control group will be assigned to a patient from the UC-MSC group once they meet all matched criteria based on age ( \pm 5 years), gender and COPD severity classification (GOLD 2019). AEs, adverse events; COPD, chronic obstructive pulmonary disease; GOLD, Global Initiative for Chronic Obstructive Lung Disease; SAEs, serious adverse events; UC-MSC, umbilical cord-derived mesenchymal stem/stromal cells.

\section{Sample size}

As a previous study indicated that the $\mathrm{FEV}_{1}(\%)$ of patients with COPD was reduced to $35.4 \% \pm 7.1 \%$ (6\% reduction) after 6 months postadministration, we set this indicator at $18 \%$ reduction after 12 months postadministration to calculate the minimum sample size for the proposed study. ${ }^{1821}$ According to the continuous endpoint of two independent sample studies, ${ }^{22}$ we assumed $\alpha$ was 0.05 and type-II error $\beta$ was 0.2 ; thus, the smallest sample size was 40 patients. The calculated sample size was 20 for each group.

\section{Matching strategy}

The patients from the control group will be assigned to a patient from the MSC group once they meet all matched criteria based on age ( \pm 5 years), gender and COPD severity classification (GOLD 2019). Patients from both groups will receive standard COPD medication management according to their COPD severity classification and based on the Vietnam Ministry of Health guideline for COPD treatment, as shown in figure 2 and table 1 .

A total of 40 patients will be recruited and assigned to the UC-MSC administration group (20 patients) and the control group ( 20 patients). The trial contains two phases: (1) the first phase will include recruiting and evaluating the first five patients from each group to assess the safety of UC-MSC administration after 1 month of follow-up, and (2) the second phase will be initiated after the first phase safety report is approved by the Ethical Committee of Vinmec International Hospital and Vietnam Ministry of Health to start recruiting the remaining 15 patients from each group to evaluate both safety and efficacy of the treatment.

\section{Participants}

The principal investigators, research and clinician team members are responsible for the study design, patient screening, recruitment, conduct and perform follow-up assessments in the trial. Participants will be allowed to enrol or withdraw at any time throughout the study. The participants will have all screening and testing costs related to the trials waived except for the costs of COPD medications or drugs. All participants' information will be protected by coding and restricted access using a computer-based system. Participants will be enrolled in the study once they meet all inclusion and exclusion criteria.

\section{Inclusion and exclusion criteria}

The diagnostic criteria and severity classification of COPD refers to the criteria established by the COPD 2019 guidelines. ${ }^{20}$ Patients will be asked to confirm the COPD conditions and classification from national hospitals and send the results to the administration office prior to enrolment in the trial for prescreening. Patients will be enrolled in the study in compliance with the inclusion and exclusion criteria established by a screening protocol as presented below.

\section{Inclusion criteria}

- Diagnosed with COPD with stage B, C or D according to GOLD 2019.

- Age between 40 and 75 years old.

- Both genders.

Exclusion criteria

- Smoker or less than 6 months of smoking cessation time.

- Asthma and other pulmonary-related diseases and injuries (including lung tuberculosis, restrictive lung disease, idiopathic pulmonary fibrosis or lung cancer).

- Acute and/or active infection.

- Cancer.

- Patients with complex cardiovascular diseases (including valvular heart disease, cardiomyopathy, arrhythmia, congenital heart disease, hypertrophy syndrome).

- Liver and kidney failure.

- Pregnancy.

- Patients with life expectancy less than 6 months due to concomitant illness.

- Under immunosuppressive treatment within 8 weeks of the first screening visit.

- Patient diagnosed diabetes with Haemoglobin A1C $\left(\mathrm{HbA}_{1} \mathrm{C}\right)>7 \%$.

\section{Recruitment}

Patients can only enrol in this study after passing the prescreening process, consultation resolution and signing the informed consent form.

The recruitment campaign will target three main sources. First, potentially eligible hospitalised patients diagnosed with severe COPD will be approached and 


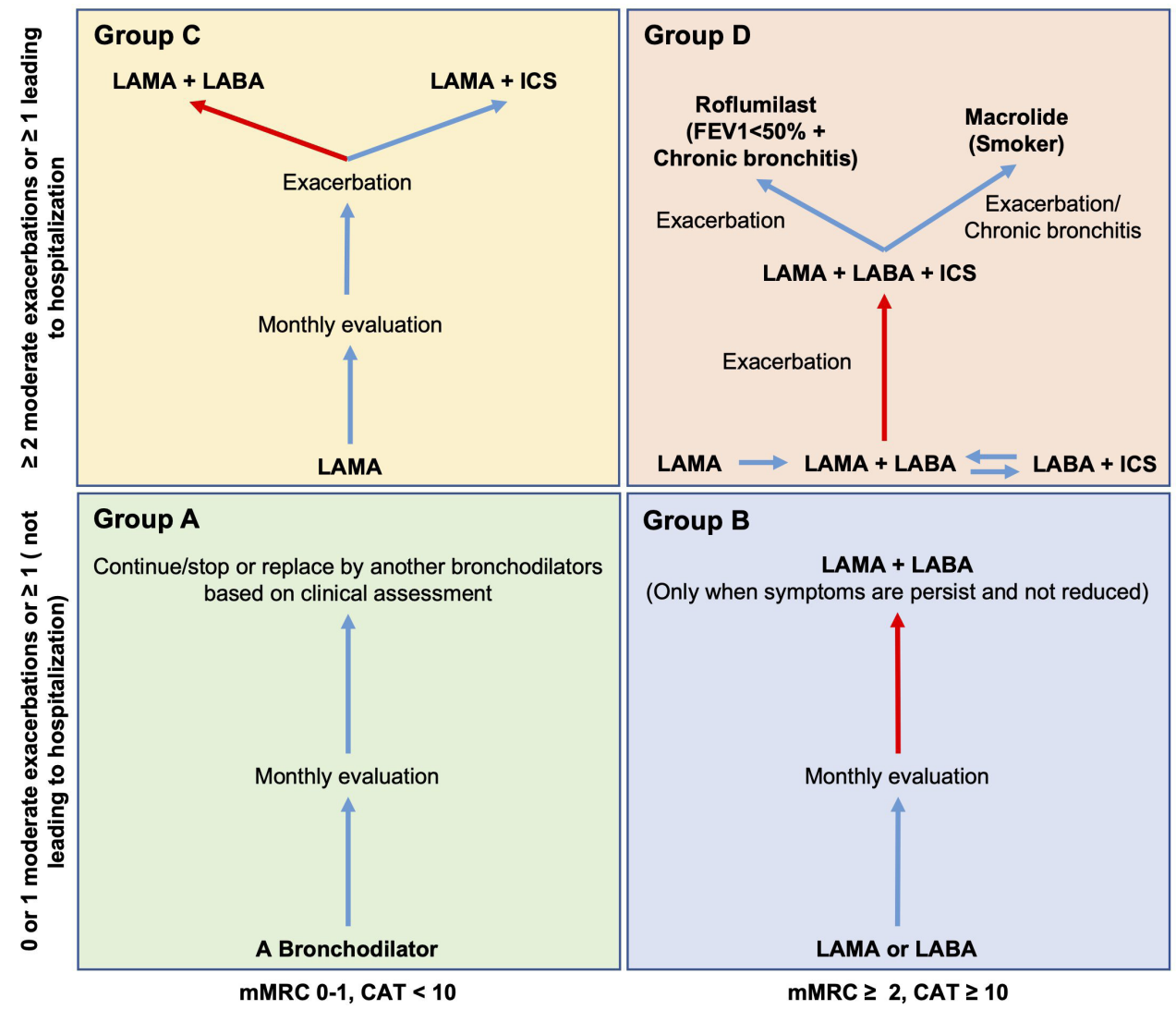

Figure 2 Standard COPD medication treatment for both groups according to GOLD 2019 and Vietnam Ministry of Health Guideline. Matched patients with COPD will be treated using the same treatment based on their GOPD 2019 classification (groups A, B, C and D). Group A (not included in this study): a single bronchodilator will be used and based on the clinical assessments and persistence of the symptoms to continue/stop or replace by another bronchodilator. Group B: single LAMA or LABA will be initially used. If the symptoms are not reduced, a combination of both LAMA and LABA will be applied. Group C: a single LAMA drug will be used for initial treatment. If exacerbations occur, LAMA and LABA combination will be applied as priority. The LAMA+ICS will be applied in specific cases based on clinical assessment, as the ICS has been reported to have severe side effects on lung inflammation. Group D: Should start the treatment with LAMA. If the patient has CAT >20, LABA and LAMA will be used as initial treatment. LABA+ICS will be used as the initial treatment only when the patient has asthma COPD overlap or the patient's eosinophil level $>300$. If exacerbation occurs after the initial treatment, the combination of LAMA, LABA, and ICS should be applied. Additional roflumilast should be used if $\mathrm{FEV}_{1}<50 \%$ and the patient has chronic bronchitis. Macrolide should be used if the patient is a former smoker. The red arrow indicates priority treatment. COPD, chronic obstructive pulmonary disease; GOLD, Global Initiative for Chronic Obstructive Lung Disease; ICS, inhaled corticosteroid; LABA, longacting $\beta$ agonists; LAMA, long-acting muscarinic antagonists; m-MRC, modified medical research council.

asked to participate in the study. Second, physicians will generate lists of patients from the electronic medical system of Vinmec Times City International Hospital with a diagnosis of COPD based on severity classification matching the GOLD 2019 criteria who were discharged within 2 years. Investigators or physicians will contact patients by telephone or mail them a research leaflet and recruitment letter. Third, leaflet and trial recruitment letters will be posted in the Vingroup cooperation internal email system, the official website, and the Facebook public platform of the Vinmec Healthcare system for those diagnosed with COPD GOLD 2019 (B, C, D) at other hospitals. If the patients are interested in this research, we will ask them to send the prescreen results to the administration office.

A multidisciplinary consultation will be held to evaluate the prescreening results from participants to confirm whether these potential participants meet the general diagnostic criteria of COPD, including inclusion and exclusion criteria. The consultation includes physicians and experts from respiratory, radiology, laboratory and stem cell biology fields. If more than $80 \%$ of experts agree on the prescreening results, patients will be viewed as potential participants. The researchers will set an appointment to communicate with the potential candidates about the clinical trial details, including pros and cons of stem cell treatments and sign the written informed consent form prior to assigning patients to either stem cell administration or control groups.

The details of the clinical trial will be explained to patients by investigators or physicians as follows: (1) the study aims and scope, (2) background of COPD and UC-MSC, (3) number of participants, study duration, and classification into either MSC administration or 
Table 1 Standard medication treatment for both groups based on GOLD 2019 guidelines and Vietnam Ministry of Health recommendations

\begin{tabular}{llll}
\hline Items & $\begin{array}{l}\text { COPD GOLD } \\
\text { 2019 group B }\end{array}$ & $\begin{array}{l}\text { COPD GOLD } \\
\text { 2019 group C }\end{array}$ & $\begin{array}{l}\text { COPD GOLD } \\
\text { 2019 group D }\end{array}$ \\
\hline Initial treatment & $\begin{array}{l}\text { A long acting } \\
\text { bronchodilator } \\
\text { (LABA or } \\
\text { LAMA) }\end{array}$ & LAMA & LAMA \\
$\begin{array}{l}\text { Difficulty in } \\
\text { breathing } \\
\text { (moderate) }\end{array}$ & LAMA +LABA & $\begin{array}{l}\text { LAMA +LABA } \\
\text { Or LAMA +ICS }\end{array}$ & $\begin{array}{l}\text { LAMA +LABA } \\
\text { ICS/LABA use } \\
\text { when: }\end{array}$ \\
& & $\begin{array}{l}\text { Asthma COPD } \\
\text { overlap. } \\
\text { Eosinophils } \\
>300 / \text { LL. }\end{array}$ \\
\hline $\begin{array}{l}\text { Difficulty in } \\
\text { breathing (Severe) }\end{array}$ & LAMA+LABA & $\begin{array}{l}\text { LAMA +LABA } \\
\text { Or LAMA +ICS }\end{array}$ & \begin{tabular}{l} 
LAMA+LABA+ICS \\
\hline
\end{tabular}
\end{tabular}

Name of Drugs use in Standard COPD Medication Treatment for both groups

\begin{tabular}{|c|c|}
\hline SABA & Salbutamol, Terbutaline, Fenoterol \\
\hline LABA & Indacaterol, Bambuterol \\
\hline SAMA & Ipratropium \\
\hline LAMA & Tiotropium \\
\hline SABA+SAMA & $\begin{array}{l}\text { Ipratropium and salbutamol } \\
\text { Ipratropium and fenoterol }\end{array}$ \\
\hline LABA+LAMA & $\begin{array}{l}\text { Indacaterol and Glycopyronium } \\
\text { Olodaterol and Tiotropium } \\
\text { Vilanterol and Umeclidinium }\end{array}$ \\
\hline ICS +LABA & $\begin{array}{l}\text { Budesonid and Formoterol } \\
\text { Fluticason and Vilanterol } \\
\text { Fluticason and Salmeterol }\end{array}$ \\
\hline Antibiotics & $\begin{array}{l}\text { Erythromycin } \\
\text { Rofumilast* }^{\star}\end{array}$ \\
\hline $\begin{array}{l}\text { Long/short- } \\
\text { acting Xanthine }\end{array}$ & Theophyllin/Theostat \\
\hline
\end{tabular}

*Roflumilast was used only when patients' FEV1 $<50 \%$ and had at least 1 admission within 1 year.

COPD, chronic obstructive pulmonary disease; GOLD, Global Initiative for Chronic Obstructive Lung Disease; ISC, inhaled corticosteroid; LABA, longacting $\beta$ agonists; LAMA, long-acting muscarinic antagonists; SAMA, Shortacting muscarinic antagonist.

control group, (4) study procedure (including screening, COPD medication management, follow-up tests), (5) potential discomfort and risks of MSC administration (including prespecified adverse events (AEs) and severe AEs (SAEs), (6) expected outcomes of the treatment (primary safety evaluation and potential therapeutic improvement of both MSC administration and COPD medication management according to Vietnam Ministry of Health guideline), (7) protection policy of patients' information and privacy and (8) voluntary participation (right and responsibility of patients). Patients will only sign written informed consent when all the above items are fully explained and the patients fully understand the protocol. The patients' baseline characteristics will be assessed by the clinicians within 30 days prior to UC-MSC administration for patients in the MSC administration group (table 2).

\section{Intervention}

Thirty UC samples were obtained from healthy women with an uncomplicated, at term pregnancy who underwent serological testing, including tests for HIV, cytomegalovirus, Epstein-Barr virus, hepatitis A virus, hepatitis $\mathrm{B}$ virus, hepatitis $\mathrm{C}$ virus, syphilis, and chlamydia, at 38 weeks of pregnancy, as shown in previous study. ${ }^{23}$ The UC tissues were collected at delivery and transferred to the Stem Cell Core Facility at the Vinmec Research Institute of Stem Cell and Gene Technology under ISO 14644-1 (certification number: CR61119-1). To generate a UC-MSC line for the current study, a single eligible UC tissue will be processed, isolated and cultured under xenofree and serum-free conditions as previously described. ${ }^{23}$ UC-MSCs will be expanded under these conditions to passage 5 (P5) and cryopreserved in the serum- and xeno-free defined reagent CryoStore CS10 (Stem Cell Technology, Canada) in liquid nitrogen (gas phase) in an automated Brooks System (Brooks Life Science, USA) for long-term storage. The releasing criteria for UC-MSC products are shown in table 3 .

To prepare UC-MSCs for therapy, aliquots of P5 UC-MSCs will be thawed in a temperature control water bath or incubator on the infusion day. The hUC-MSCs will be washed and suspended in $0.9 \%$ normal saline. In addition to inspecting the quality of the UC-MSC product based on the releasing criteria, the staff of the Cell Therapy Department will confirm the viability and quality of the UC-MSC product before the infusion. The cell dose will be calculated based on patients' body weight and cell viability results to obtain the dose of $1 \times 10^{6}$ viable cells/ $\mathrm{kg}$ patient body weight prior to transport to the administration ward. Currently, there is no effective treatment for patients with COPD. Thus, the intervention group will be given the standard COPD medication management as primary treatment and extra UC-MSC administration, while the control group will receive only the standard COPD treatment (table 1).

\section{Mode of cell administration (UC-MSC group)}

Patients assigned to UC-MSC administration groups will receive two administrations at a dose of 1 million cells/ $\mathrm{kg}$ patient bodyweight via the IV route with a 3-month intervening interval. On the day of infusion, thawed cells at P5 will be prepared to meet the target administration dose based on the number of viable cells in $10 \mathrm{~mL}$ of $0.9 \%$ Sodium chloride $(\mathrm{NaCl})$ (Braun, USA) as described above and delivered to the administration ward for infusion at a rate of $20 \mathrm{~mL} /$ hour.

\section{Withdrawal}

Participant discontinuation may occur on participant death, SAEs, other serious disease-limiting involvement, or a direct request from participant to withdraw from the study. Once the participant withdraws from the study, the reasons for the withdrawal and all recorded results will be documented in detail. New participants will not be recruited to replace withdrawn participants. 
Table 2 Study timeline and clinical procedures during the trial

\begin{tabular}{|c|c|c|c|c|c|c|}
\hline Study procedure & Prescreening & Screening phase* & Baseline & 3 months & 6 months & 12 months \\
\hline UC-MSC administration† & & & $\nabla$ & $\nabla$ & & \\
\hline Medication treatment $\neq$ & & & $\nabla$ & $\nabla$ & $\nabla$ & $\nabla$ \\
\hline Inclusion and exclusion criteria & & $\nabla$ & & & & \\
\hline Demographic information & & $\nabla$ & $\nabla$ & & & \\
\hline Vital signs§/physical examination & & $\nabla$ & $\nabla$ & $\nabla$ & $\nabla$ & $\nabla$ \\
\hline COPD assessment & $\nabla$ & $\nabla$ & & & & \\
\hline COPD GOLD 2019 classification & $\nabla$ & $\nabla$ & & $\nabla$ & $\nabla$ & $\nabla$ \\
\hline Haematology analysis & $\nabla$ & $\nabla$ & $\nabla$ & $\nabla$ & $\nabla$ & $\nabla$ \\
\hline $\begin{array}{l}\text { Infectious disease examination/ } \\
\text { test }^{\star \star}\end{array}$ & $\nabla$ & $\nabla$ & $\nabla$ & & & \\
\hline Chest CT scan & & $\nabla$ & $\nabla$ & & $\nabla$ & $\nabla$ \\
\hline Chest X-ray & & $\nabla$ & $\nabla$ & & $\nabla$ & $\nabla$ \\
\hline Pulmonary function analysis & & $\nabla$ & $\nabla$ & $\nabla$ & $\nabla$ & $\nabla$ \\
\hline Adverse event evaluation & & & $\square$ & $\nabla$ & $\nabla$ & $\square$ \\
\hline Mortality/complications monitoring & & & $\nabla$ & $\nabla$ & $\nabla$ & $\nabla$ \\
\hline
\end{tabular}

*If the results of the screening phase for UC-MSC groups are within 30 days of UC-MSC administration, they will be automatically considered as the baseline level.

†Applies only for the UC-MSC group at baseline and 3 months.

$\ddagger$ Treatment medication applies for all testing groups based on patients’ COPD classification according to GOLD 2019 guidelines.

$\S$ Vital signs include body temperature, blood pressure, heart rate, respiratory rate, oxygen saturation and patient body weight.

१Haematological analysis included white cell count, platelet count, red cell count, haemoglobin, percentage of lymphocytes, neutrophils, monocytes, eosinophils, basophils, $\mathrm{C}$ reactive protein, pro-BNP and troponin-T and D-dimer.

${ }^{* *}$ Infectious diseases include hepatitis, syphilis, HIV, HBV and tuberculosis.

††Blood gas analysis includes $\mathrm{pH}, \mathrm{PaO}_{2}, \mathrm{PaCO}_{2}, \mathrm{BE}, \mathrm{HCO}_{3}-$.

COPD, chronic obstructive pulmonary disease; GOLD, Global Initiative for Chronic Obstructive Lung Disease; HBV, hepatitis B virus; UC-

MSC, umbilical cord-derived mesenchymal stem/stromal cells.

\section{Adverse events}

AEs are defined as adverse medical events that occur after the patient signs informed consent until completion of the follow-up period. AEs include abnormal laboratory results, symptoms, or diseases. All AEs will be documented on a written case report form (CRF) and transferred to a Research Electronic Data Capture (RedCap) system. Once AEs occur, the physician and clinician in charge will follow the necessary treatment according to the patient's condition and decide whether to suspend clinical research. In terms of SAEs, the clinician team will follow the first priority to treat principle and be considered an emergency situation. The principal investigators will immediately inform the Ethical Committee and Medical Advisory board of Vinmec Times City International Hospital. Within 24 hours, the SAE report should be submitted with full description, while a follow-up SAE report should be submitted to the Ethical Committee of Vinmec Times City International Hospital. Within the 7 days, the SAE report with comments from the Ethical Committee will be submitted to the National
Ethical Committee of Vietnam Ministry of Health via post. All participants enrolled in the study will be subjected to an insurance policy that provides ancillary and posttrial medical care in case of injury or death as a result of their participation in the trial.

\section{Outcome evaluation}

Primary outcomes (safety)

All required evaluation and laboratory tests with the timeframes are listed in table 1 . To assess safety, the number of AEs or SAEs during stem cell administration (72 hours) at 3 months, 6 months and 12 months after discharge will be evaluated. Body temperature, blood pressure, respiratory rate, heart rate and saturated oxygen level inblood $\left(\mathrm{SpO}_{2}\right)$ will be recorded in real-time before and during MSC administration up to 24 hours. Additionally, D-dimer level and patients' blood analysis will be performed at administration and 24 hour postinfusion to monitor the potential thrombotic events as previously reported..$^{24}$ As mentioned above, the first phase of this study will involve recruiting five pairs of patients to evaluate the safety prior 
Table 3 Release criteria and stem cell quality control

\begin{tabular}{|c|c|c|}
\hline Criteria & Testing method & $\begin{array}{l}\text { Released } \\
\text { criteria }\end{array}$ \\
\hline \multicolumn{3}{|l|}{$\begin{array}{l}\text { Positive markers (\%) } \\
\text { (median, range) }\end{array}$} \\
\hline CD73 & \multirow{4}{*}{$\begin{array}{l}\text { Flow cytometry using } \\
\text { the Human MSC } \\
\text { Analysis Kit (Becton } \\
\text { Dickinson,USA) }\end{array}$} & $>95 \%$ \\
\hline CD90 & & $>95 \%$ \\
\hline CD105 & & $>95 \%$ \\
\hline $\begin{array}{l}\text { Negative markers } \\
(\%)\end{array}$ & & $<2 \%$ \\
\hline $\begin{array}{l}\text { Cell viability }(\%) \\
(\text { mean } \pm \text { SD) }\end{array}$ & Trypan blue staining & $>80 \%$ \\
\hline Microorganism tests & $\begin{array}{l}\text { BacT/Alert 3D } \\
\text { microbial detection } \\
\text { system (Biomerieux, } \\
\text { USA) }\end{array}$ & Negative \\
\hline Mycoplasma & $\begin{array}{l}\text { MycoAlertTM plus } \\
\text { mycoplasma detection } \\
\text { kit (Lonza, Switzerland) }\end{array}$ & Negative \\
\hline Endotoxin & $\begin{array}{l}\text { Endosafe-PTS (Charles } \\
\text { river laboratories) }\end{array}$ & $\leq 5 \mathrm{EU} / \mathrm{kg}$ \\
\hline $\begin{array}{l}\text { Immunoregulatory } \\
\text { assay }\end{array}$ & Flow cytometry & $\begin{array}{l}\text { Not } \\
\text { Applicable }\end{array}$ \\
\hline
\end{tabular}

To assess the quality of UC-MSCs for administration, a set of release criteria was defined, which included the following: the positive markers (CD73, CD90 and CD105) must be higher than 95\%, the negative markers (CD11b, CD19, CD34, CD45 and HLA-DR) must be less than $2 \%$; the cell viability must be higher than $80 \%$ with a normal karyotype; and the cell product must be free from microorganism infections and mycoplasma. Immunoregulatory assays will be performed to assess but not consider released criteria.

HLA-DR, Human Leukocyte Antigen - DR isotype; UC-MSC, umbilical cord-derived mesenchymal stem/stromal cells.

to initiating the second phase. The safety report of the first phase will cover 1-month postdischarge and will be submitted to the Ethical Committee of Vinmec Times City International Hospital and the National Ethical Committee of Vietnam Ministry of Health for approval of starting the second phase.

SAEs include death, any critical cardiac event (new ventricular tachycardia, ventricular fibrillation, or asystole, cardiac arrest, cardiac hypertrophy), acute pulmonary distress and embolism, stroke, anaphylactic shock, sepsis and other conditions that extend the hospital stay. The prespecified AEs include fever, common allergic reactions (rash, oedema, erythema, pallor), infection at the administration site, changes in vital signs and abnormal laboratory test results (including haematological analysis and indicators of liver and kidney functions).

\section{Secondary outcomes (efficacy)}

The efficacy endpoints are as follows: (1) number of admissions and readmissions, (2) general self-efficacy, (3) the number of admissions and unscheduled outpatient visits due to symptoms of COPD, (4) arterial blood gas analysis (including $\mathrm{pH}$, partial pressure of oxygen $\left(\mathrm{PaO}_{2}\right)$, partial pressure of carbondioxide $\left(\mathrm{PaCO}_{2}\right)$, base excess $(\mathrm{BE})$, bicarbonate $\left(\mathrm{HCO}_{3}-\right),(5)$ respiratory functions (Forced Expiratory Volume in 1 second $\left(\mathrm{FEV}_{1}\right)$, Tiffeneau-Pinelli index $\left(\mathrm{FEV}_{1} / \mathrm{FVC}\right)$, Vital capacity (VC), Total Lung capacity (TLC), Residual volume (RV), Diffusing capacity for carbon monoxide (DLCO), Diffusing capacity for nitric oxide and carbon monoxide (DLNO/DLCO), (6) electrocardiogram (ECG), echocardiography, high-resolution chest CT, abdominal ultrasound, abnormality of thyroid and mammary gland, (7) inflammatory response $(\mathrm{C}$ reactive protein $(\mathrm{CRP})$, pro B-type natriuretic peptide (pro-BNP) and troponin-T) and (8) cytokine analysis from patients' plasma. In addition, the modified medical research council (m-MRC) questionnaire and quality of life (St. Georges Respiratory Questionnaire (SGRQ)) will be used to monitor respiratory function improvement. To reveal the therapeutic effects of UC-MSC administration, UC-MSC characterisation will be conducted in vitro, including MSC marker analysis, metabolic evaluation, immunoregulatory assessment and cytokine secretion analysis (table 4).

\section{Follow-up procedure}

Follow-up visits will be conducted at 3, 6 and 12 months after hUC-MSC administration. Patients will be asked to come to the hospital to undergo an assessment of their conditions according to the protocol procedure. The safety follow-up will include an extra 1-month follow-up point via telephone and outpatient contact, and patients will only be asked to make an appointment if AEs or SAEs occur.

\section{Data collection}

The data accumulated during the trial will be documented in the patients' medical records and the CRF. The quality control officers from Vinmec Times City International Hospital and Vinmec Scientific Research Board independently checked the accuracy and consistency of the CRF data with the original patients' medical records to ensure that the data were accurately entered into the CRF. Once the CRF is checked, within 7 days, all data will be recorded to RedCap software by assigned personnel and crosschecked by principle investigators. There are four data collection points, including baseline and 3 months, 6 months and 12 months postadministration. The internal auditor of the Vinmec Research Institute of Stem Cell and Gene Technology will review each original research record to confirm the accuracy, consistency, timely records and meet the standard requirements. Data analysis will be performed using RedCap and statistical analysis software following the statistical analysis strategy (https://redcap.vinmec.com/). The data of this clinical trial will be disseminated with permission from funding bodies and principal investigators through national and international conferences, peer-reviewed publications and scientific reports. A complete data set will be available on request after trial completion. 
Table 4 Proposed experiments design for evaluation the potential therapeutic mechanism of UC-MSCs in the treatment of COPD

\begin{tabular}{|c|c|c|c|}
\hline $\begin{array}{l}\text { Proposed } \\
\text { experiments }\end{array}$ & Criteria & Cell type/method & Expected outcomes \\
\hline \multirow{3}{*}{$\begin{array}{l}\text { UC-MSC } \\
\text { characterisation }\end{array}$} & MSC marker analysis & UC-MSCs/flow cytometry & Meet ISCT guideline \\
\hline & Karyotype & UC-MSCs/G-banding method & Normal post-expansion \\
\hline & $\begin{array}{l}\text { Growth factor, } \\
\text { cytokines secretion }\end{array}$ & $\begin{array}{l}\text { UC-MSCs/ProcartaPlex } \\
\text { Immunoassays }\end{array}$ & $\begin{array}{l}\text { Detection of cytokines and growth factors } \\
\text { involves in anti-inflammatory and tissue } \\
\text { regeneration process. }\end{array}$ \\
\hline \multirow[t]{2}{*}{$\begin{array}{l}\text { Metabolic } \\
\text { evaluation }\end{array}$} & $\begin{array}{l}\text { Mitochondrial } \\
\text { activities }\end{array}$ & $\begin{array}{l}\text { UC-MSCs/agilent seahorse XF } \\
\text { cell mito stress test }\end{array}$ & $\begin{array}{l}\text { Measurement of mitochondrial activities of UC- } \\
\text { MSCs pre-administration }\end{array}$ \\
\hline & Glycolysis & $\begin{array}{l}\text { UC-MSCs/agilent seahorse XF } \\
\text { glycolysis stress test }\end{array}$ & $\begin{array}{l}\text { Measurement of glycolysis process of UC-MSC } \\
\text { pre-administration }\end{array}$ \\
\hline $\begin{array}{l}\text { Immunoregulatory } \\
\text { assessment }\end{array}$ & & $\begin{array}{l}\text { UC-MSCs+peripheral } \\
\text { mononuclear cells from patients } \\
\text { with COPD }\end{array}$ & $\begin{array}{l}\text { UC-MSCs inhibit the proliferation rate of } \\
\text { lymphocytes in the present of PHA in a similar } \\
\text { manner to healthy donor counterpart }\end{array}$ \\
\hline \multirow[t]{3}{*}{$\begin{array}{l}\text { Growth factors } \\
\text { and cytokines } \\
\text { analysis }\end{array}$} & $\begin{array}{l}\text { UC-MSC secretion } \\
\text { profiles under xeno- } \\
\text { free and serum-free } \\
\text { culture conditions }\end{array}$ & $\begin{array}{l}\text { UC-MSCs/procartaPlex } \\
\text { immunoassays }\end{array}$ & $\begin{array}{l}\text { Detection of cytokines and growth factors } \\
\text { involves in anti-inflammatory and tissue } \\
\text { regeneration process. }\end{array}$ \\
\hline & $\begin{array}{l}\text { Cytokine profiles from } \\
\text { patients with COPD } \\
\text { plasma }\end{array}$ & $\begin{array}{l}\text { Patients with COPD plasma/ } \\
\text { procartaPlex immunoassays }\end{array}$ & $\begin{array}{l}\text { Evaluation of cytokines involves in inflammatory } \\
\text { response obtain from patients with COPD plasma } \\
\text { before and after UC-MSC administration. }\end{array}$ \\
\hline & $\begin{array}{l}\text { Interaction between } \\
\text { UC-MSCs and } \\
\text { patients with COPD' } \\
\text { lymphocytes }\end{array}$ & $\begin{array}{l}\text { Media obtained from co-culture } \\
\text { of UC-MSCs and peripheral } \\
\text { mononuclear cells from patients } \\
\text { with COPD/ProcartaPlex } \\
\text { Immunoassays }\end{array}$ & $\begin{array}{l}\text { Detection of cytokines involves in the anti- } \\
\text { inflammatory functions of UC-MSCs. }\end{array}$ \\
\hline
\end{tabular}

COPD, chronic obstructive pulmonary disease; GOLD, Global Initiative for Chronic Obstructive Lung Disease; ISCT, International Society for Cellular Therapy.

\section{Statistical analysis strategy}

Descriptive statistics will be used to illustrate the demographics of the patients with COPD. Categorical variables are expressed as proportions, whereas quantitative variables are described as the mean values and their SD or as the medians and their IQRs. The number and type of AEs/SAEs will be compared between the two treatment groups using the $\chi^{2}$ (or Fisher's exact) test. For the intervention and control groups, the indicators (m-MRC, COPD Assessment Test (CAT), SGRQ, respiratory functions, and arterial blood gas analysis) at baseline and at 3 months, 6 months and 12 months will be compared with repeated measures Analysis of variance. $\mathrm{P}<0.05$ will be considered statistically significant. The analyses will be performed using Stata V.14 (StataCorp).

\section{Patient and public involvement}

The patients and public were not involved in the design, or conduct, or reporting or dissemination plans of our research.

\section{ETHICS AND DISSEMINATION}

This trial, including the consent form and clinical protocol, was approved by the Ethical Committee of Vinmec International Hospital (number: 166/2019/ QĐ-VMEC) and Vietnam Ministry of Health (number: 2002/QĐ-BYT). The trial conforms with the Declaration of Helsinki. All participants will provide oral and written informed consent prior to participating in the study. This study will be reported in accordance with the STROBE guidelines for matched case-control trial. ${ }^{25}$ We will disseminate the research results through highquality peer-reviewed open access (via PubMed) journals and presentations at national and international conferences. Finally, an ongoing update of the trial will also be provided and shared annually with our partners in the health system and community agencies according to the National Regulation. 


\section{DISCUSSION}

This study protocol presents the matched-control phase I/II clinical trial evaluating the safety and potential efficacy of allogeneic UC-MSC administration in patients with moderate-to-severe COPD (GOLD 2019). To date, there is no effective treatment available for patients with COPD and pharmacological interventions are hampered by the heterogeneity of disease mechanisms and phenotypic alternation. Therefore, establishing new treatment methods to reduce the devastating effects of COPD is imperative. The body of preclinical studies and human clinical trials suggests that MSC administration emerges as a potential therapeutic approach for COPD because MSCs have been found to be well tolerated and safe in many clinical trials and have proven their effectiveness in animal models. Several clinical trials have been conducted in COPD. Most of these studies were phase 1 safety trials, which uniformly reported no obvious adverse events and serious adverse events as well as no evidence of infusional toxicities during the follow-up period. ${ }^{26}$ However, the effectiveness of MSC therapy showed differences among various clinical trials, and a small number of trials have revealed no significant changes in lung function and fibrosis postadministration compared with baseline levels. ${ }^{27}$ Therefore, it is important to comprehensively analyse the factors that directly contribute to treatment safety and efficacy.

Human clinical trials were conducted to evaluate the safety and efficacy of MSCs in the treatment of COPD, including five studies using bone marrow-derived cells $^{14-1719}$ and a pilot study using UC-MSCs. ${ }^{18}$ The first study supported the safety profile of MSCs administered BMMCs to four patients with COPD, although the overall clinical outcomes did not demonstrate the efficacy of the treatment. It is understandable that studies together with the two trials (NCT001110252 and NCT01306513) are phase 1 clinical trials that aimed to evaluate the safety and feasibility of cellular administration in the treatment of COPD. Notably, the NCT001110252 study followed up with patients for up to 3 years illustrated an overall reduction in the process of COPD pathological development. ${ }^{19}$ In a pilot study using UC-MSCs, patients with COPD were followed up for 6 months, and no AEs or SAEs were observed throughout the course of the study. Although clinical outcomes such as COPD exacerbations, m-MRC score and CAT were significantly reduced postadministration, pulmonary function parameters remained unchanged compared with baseline. ${ }^{18}$ In our current study, we use UC-MSCs as an 'off-the-shelf' product for administration, providing flexibility in patient management and standardised products for all treated patients, allowing more accuracy in evaluation. Moreover, by using a matched control design, our study aims to eliminate the variability in COPD conditions between the intervention and control groups to accurately evaluate the safety and efficacy of the treatment. In general, it was confirmed that MSC administration is well tolerated without serious adverse events or administration-associated AEs and is not associated with significant alterations in spirometry, immune function, cardiovascular activity or patient quality of life. ${ }^{28}$

In both preclinical models of COPD and clinical trials, MSCs regardless of their sources exhibit their therapeutic potential via either anti-inflammatory paracrine actions, reducing the rate of pulmonary fibrosis and/or lung recovery. In rodent models, studies using BM-MSCs or adipose-derived MSCs (AD-MSCs) have demonstrated that these cells administered via intravenous injection or intra-tracheal instillation were safe and effective in attenuating airway injury and enhance the recovery of lung functions via reducing airway inflammation and apoptosis. ${ }^{29}$ In mice model, administration of UC-MSCs (from Wharton's Jelly) significantly improved the pulmonary function and regeneration in COPD-induced mice compared with the sham group. ${ }^{30}$ To date, no comparative study is conducted to address the differences in efficacy introduce by MSCs from different sources. In our previous study, we demonstrated that MSCs from perinatal and adult sources behaved differently even when they were cultured under a standardise culture platform (xeno-free and serum-free). ${ }^{23}$ Therefore, although it seems that MSCs derived from different sources might show similar effects on COPD-induced animal models, we believe that the source of MSCs might play a role in the level of the therapy effectiveness and their mechanism of action might also different, especially when they are exposed to COPD-related microenvironment.

To provide an insight into the mechanism of action of MSC administration in response to COPD conditions, this study aims to evaluate the response of patients' lymphocyte to UC-MSC in vitro by cocultures patients with COPD lymphocytes (before-and-after UC-MSCs administration at different timepoints) with UC-MSCs to evaluate the potential effect of UC-MSCs on patient's lymphocytes compare to that of healthy donors. Hence, this experiment is not only evaluating the UC-MSC potency but also reveal the potential mechanism of MSC actions in patients with COPD. We expect the UC-MSCs would inhibit the proliferation of COPD patient's lymphocytes in a similar manner to that of healthy donor. The culture media of UC-MSC alone, lymphocyte alone, and co-culture of UC-MSCs and lymphocyte will be subjected to cytokines analysis of inflammatory factors such as Interleukin- $1 \beta$ (IL-1 $\beta$ ), Tumor necrosis factor- $\alpha$ (TNF- $\alpha)$, IL-4, IL-8, IL-10, etc to identify the release of soluble mediators from UC-MSCs that might involve in reducing lung inflammation. Toward this aim, we speculate the potential mechanism of MSC actions for COPD includes: (1) reduction of inflammatory reactions at injured airway via either paracrine effects or cell-to-cell contact with immune cells, (2) reduction of pulmonary fibrosis and airway thickening process and (3) improvement of parenchymal repair by secretion of wide range of cytokines and growth factors.

The major delivery routes of MSCs in the treatment of pulmonary disease include intraperitoneal (usually in animal models), intranasal or intratracheal and 
intravenous administration. The intratracheal administration of MSCs was performed in children with BPD in several small uncontrolled studies. However, in terms of COPD, all trials used intravenous administration with the aim of investigating whether systemic administration of MSCs is safe and effective in patients with COPD. In fact, the intravenous administration route is considered a better option compared with intratracheal delivery for several reasons. Previous studies illustrated that intravenous administration of MSCs was safe and potentially provided therapeutic effects in several lung diseases, including COPD. ${ }^{15} 27$ Moreover, a systemic analysis of preclinical studies suggested that intravenous administration of MSCs introduced better effects than those administered via the intratracheal route. ${ }^{13}$ We hypothesised that the results of this clinical trial will provide data supporting that UC-MSC administration via the intravenous route is safe, feasible, and potentially effective in patients with COPD.

The dose-escalating evaluation has been conducted in several clinical trials for various diseases, including pulmonary syndromes, using a wide range of UC-MSC doses from 0.5 to 10 million cells $/ \mathrm{kg}$ via intravenous administration. ${ }^{152731}$ Notably, limited studies have reported the different effects of MSC doses in patients with COPD. In fact, a relatively high dose ( 10 million cells $/ \mathrm{kg}$ patient body weight) was tested in patients with ARDS without any administration-associated AEs or SAEs recorded. However, it is important to note that delivery of a high dose of stem cells might increase the risk of pulmonary embolism and thrombosis regardless of administration route, which was demonstrated previously in animal models and clinical trials. ${ }^{32-34}$ Therefore, in this trial, we used the most common dose of MSCs used in numerous studies, which is 1 million cells $/ \mathrm{kg}$ patient body weight.

In preclinical models of elastase-induced emphysema, two doses of MSCs improved anti-inflammatory regulation and lung recover compared with a single dose of MSCs. ${ }^{35}$ In clinical trial using BM-MSCs, patients with COPD received four infusions showed a reduction in circulating CRP and in combination with lung volume reduction strategy, two infusions were enough to increase the expression of CD31, an indication of microvascular endothelial cell response. ${ }^{17}{ }^{26}$ Moreover, the effects of autologous MSC administration were reported to be relatively narrow because it was reported the positive effects in patients with type 2 diabetes were observed as early as 1-month postadministration of MSCs and started to diminish after 3-6months postadministration. ${ }^{36} 37$ Hence, in this current study, we will perform two doses of UC-MSCs with a 3-month intervening interval.

This clinical trial has several advantages. First, this is the first trial using an 'off-the-shelf' product (UC-MSCs) for patients with COPD. Second, this is the first trial to investigate the therapeutic effects of UC-MSCs as supplementary products in combination with standard medication treatments according to the GOLD 2019 recommendation. Third, if the potential efficacy can be detected throughout the course of our study, our results (including MSC biological analysis of stem cell characterisation, immunoregulation and metabolism) will strengthen our knowledge and understanding of UC-MSC effects in COPD and provide a fundamental background for treating patients with moderate-to-severe COPD. In the case of no therapeutic effect, our data will also provide important insight into the safety of the treatment and potential alternative approach for MSC therapy of COPD.

\section{Author affiliations}

${ }^{1}$ Department of Research \& Development, Vinmec Research Institute of Stem Cell and Gene Technology, Vinmec Heathcare System, Hanoi, Vietnam

${ }^{2}$ Clinical Research Department, Vinmec Research Institute of Stem Cell and Gene Technology, Vinmec Healthcare System, Hanoi, Viet Nam

${ }^{3}$ Department of Internal Medicine, Vinmec Times City International Hospital, Hanoi, Viet Nam

${ }^{4}$ Vinmec Research Institute of Stem Cell and Gene Technology, Vinmec Health Care System, Hanoi, Viet Nam

\section{Twitter Bach N Nguyen @Bách Nguyễn}

Acknowledgements The authors would like to thank all patients involved in the study for their trust, understanding and willingness. We thank our collaborating clinicians at the Vinmec Health Care System for participating in this study. We also thank our colleagues at Vinmec High-tech center and Vinmec Tissue Bank for their support with the quality control and UC-MSC experiment set up.

Contributors LTN, DMH and KTN were involved in the design of the study. DMH drafted the manuscripts with critical input from LTN, KTN, AHN and BNN. LTN, AHN and BNN contributed to the standard medical treatment checklist and drug for all patients. DMH, LTN and AHN are the grant holder and project leader, respectively. All authors reviewed, edited and approved the final version of the manuscript.

Funding This work was supported by the Vingroup Scientific Research and Clinical Application Fund (Grant number: ISC.19.16).

Disclaimer The funder has no role in the analysis or preparation of this manuscript.

\section{Competing interests None declared.}

Patient and public involvement Patients and/or the public were not involved in the design, or conduct, or reporting, or dissemination plans of this research.

Patient consent for publication Not required.

Provenance and peer review Not commissioned; externally peer reviewed.

Open access This is an open access article distributed in accordance with the Creative Commons Attribution Non Commercial (CC BY-NC 4.0) license, which permits others to distribute, remix, adapt, build upon this work non-commercially, and license their derivative works on different terms, provided the original work is properly cited, appropriate credit is given, any changes made indicated, and the use is non-commercial. See: http://creativecommons.org/licenses/by-nc/4.0/.

ORCID iD

Duc M Hoang http://orcid.org/0000-0001-5444-561X

\section{REFERENCES}

1 Rabe KF, Watz H. Chronic obstructive pulmonary disease. Lancet 2017;389:1931-40.

2 GBD 2015 Chronic Respiratory Disease Collaborators. Global, regional, and national deaths, prevalence, disability-adjusted life years, and years lived with disability for chronic obstructive pulmonary disease and asthma, 1990-2015: a systematic analysis for the global burden of disease study 2015. Lancet Respir Med 2017;5:691-706.

3 Ho T, Cusack RP, Chaudhary N, et al. Under- and over-diagnosis of COPD: a global perspective. Breathe 2019;15:24-35.

4 Nguyen T-S, Nguyen TLH, Pham TTV, et al. Impact of pharmaceutical care in the improvement of medication adherence and quality of life for COPD patients in Vietnam. Respir Med 2019;153:31-7. 
$5 \mathrm{Ngo} C Q$, Phan DM, Vu GV, et al. Inhaler technique and adherence to inhaled medications among patients with acute exacerbation of chronic obstructive pulmonary disease in Vietnam. Int J Environ Res Public Health 2019;16 doi:10.3390/ijerph16020185

6 Caplan Al. Mesenchymal stem cells: time to change the name! Stem Cells Trans/ Med 2017;6:1445-51.

7 Viswanathan S, Shi Y, Galipeau J, et al. Mesenchymal stem versus stromal cells: International Society for Cell \& Gene Therapy (ISCT®) Mesenchymal Stromal Cell committee position statement on nomenclature. Cytotherapy 2019;21:1019-24.

8 Dominici M, Le Blanc K, Mueller I, et al. Minimal criteria for defining multipotent mesenchymal stromal cells. The International Society for cellular therapy position statement. Cytotherapy 2006;8:315-7.

9 Amable PR, Teixeira MVT, Carias RBV. Protein synthesis and secretion in human mesenchymal cells derived from bone marrow, adipose tissue and Wharton's jelly. Stem Cell Res Ther 2014;5:53.

10 Qin H, Zhao A. Mesenchymal stem cell therapy for acute respiratory distress syndrome: from basic to clinics. Protein Cell 2020;11:707-22.

11 Cruz FF, Rocco PRM. The potential of mesenchymal stem cell therapy for chronic lung disease. Expert Rev Respir Med 2020;14:31-9.

12 Mohammadipoor A, Antebi B, Batchinsky Al, et al. Therapeutic potential of products derived from mesenchymal stem/stromal cells in pulmonary disease. Respir Res 2018;19:218.

13 Eggenhofer E, Benseler V, Kroemer A, et al. Mesenchymal stem cells are short-lived and do not migrate beyond the lungs after intravenous infusion. Front Immunol 2012;3:297.

14 Ribeiro-Paes JT, Bilaqui A, Greco OT, et al. Unicentric study of cell therapy in chronic obstructive pulmonary disease/pulmonary emphysema. Int J Chron Obstruct Pulmon Dis 2011;6:63-71.

15 Weiss DJ, Casaburi R, Flannery R, et al. A placebo-controlled, randomized trial of mesenchymal stem cells in COPD. Chest 2013;143:1590-8.

16 de Oliveira HG, Cruz FF, Antunes MA, et al. Combined bone marrow-derived mesenchymal stromal cell therapy and oneway endobronchial valve placement in patients with pulmonary emphysema: a phase I clinical trial. Stem Cells Trans/ Med 2017;6:962-9.

17 Stolk J, Broekman W, Mauad T, et al. A phase I study for intravenous autologous mesenchymal stromal cell administration to patients with severe emphysema. QJM 2016;109:331-6.

18 Le Thi Bich P, Nguyen Thi H, Dang Ngo Chau H, et al. Allogeneic umbilical cord-derived mesenchymal stem cell transplantation for treating chronic obstructive pulmonary disease: a pilot clinical study. Stem Cell Res Ther 2020;11:60.

19 Stessuk T, Ruiz MA, Greco OT, et al. Phase I clinical trial of cell therapy in patients with advanced chronic obstructive pulmonary disease: follow-up of up to 3 years. Rev Bras Hematol Hemoter 2013;35:352-7.

20 Singh D, Agusti A, Anzueto A, et al. Global strategy for the diagnosis, management, and prevention of chronic obstructive lung disease: the gold science Committee report 2019. Eur Respir J 2019;53:1900164. doi:10.1183/13993003.00164-2019

$21 \mathrm{Kim} \mathrm{S}$, Oh J, Kim Y-I, et al. Differences in classification of COPD group using COPD assessment test (CAT) or modified medical Research Council (mMRC) dyspnea scores: a cross-sectional analyses. BMC Pulm Med 2013;13:35.

22 Hsieh FY. Sample size formulae for intervention studies with the cluster as unit of randomization. Stat Med 1988;7:1195-201.

23 Hoang VT, Trinh Q-M, Phuong DTM, et al. Standardized xeno- and serum-free culture platform enables large-scale expansion of highquality mesenchymal stem/stromal cells from perinatal and adult tissue sources. Cytotherapy 2021;23:88-99.

24 George MJ, Prabhakara K, Toledano-Furman NE, et al. Procoagulant in vitro effects of clinical cellular therapeutics in a severely injured trauma population. Stem Cells Trans/ Med 2020;9:491-8.

25 Cuschieri S. The STROBE guidelines. Saudi J Anaesth 2019;13:31-4

26 Weiss DJ. Cell-based therapy for chronic obstructive pulmonary disease. rebuilding the lung. Ann Am Thorac Soc 2018;15:S253-9.

27 Wilson JG, Liu KD, Zhuo H, et al. Mesenchymal stem (stromal) cells for treatment of ARDS: a phase 1 clinical trial. Lancet Respir Med 2015;3:24-32.

28 Zhao R, Su Z, Wu J, et al. Serious adverse events of cell therapy for respiratory diseases: a systematic review and meta-analysis. Oncotarget 2017;8:30511-23.

29 Liu X, Fang Q, Kim H. Preclinical studies of mesenchymal stem cell (MSC) administration in chronic obstructive pulmonary disease (COPD): a systematic review and meta-analysis. PLoS One 2016;11:e0157099.

30 Cho JW, Park KS, Bae JY. Effects of Wharton's jelly-derived mesenchymal stem cells on chronic obstructive pulmonary disease. Regen Ther 2019;11:207-11.

$31 \mathrm{He}$ X, Ai S, Guo W, et al. Umbilical cord-derived mesenchymal stem (stromal) cells for treatment of severe sepsis: aphase 1 clinical trial. Trans/ Res 2018;199:52-61.

32 Tanaka E, Ogawa Y, Mukai T, et al. Dose-Dependent effect of intravenous administration of human umbilical cord-derived mesenchymal stem cells in neonatal stroke mice. Front Neurol 2018;9:133

33 Kansu E. Thrombosis in stem cell transplantation. Hematology 2012;17:s159-62.

34 Jung JW, Kwon M, Choi JC, et al. Familial occurrence of pulmonary embolism after intravenous, adipose tissue-derived stem cell therapy. Yonsei Med J 2013;54:1293-6.

35 Poggio HA, Antunes MA, Rocha NN, et al. Impact of one versus two doses of mesenchymal stromal cells on lung and cardiovascular repair in experimental emphysema. Stem Cell Res Ther 2018;9:296.

$36 \mathrm{Hu} \mathrm{J}$, Li C, Wang L, et al. Long term effects of the implantation of autologous bone marrow mononuclear cells for type 2 diabetes mellitus. Endocr J 2012;59:1031-9.

37 Liu X, Zheng P, Wang X, et al. A preliminary evaluation of efficacy and safety of Wharton's jelly mesenchymal stem cell transplantation in patients with type 2 diabetes mellitus. Stem Cell Res Ther 2014;5:57. 